\title{
Investigation of the Shear Response and Geometrically Necessary Dislocation Densities in Shear Localization in High-Purity Titanium
}

Chaoyi Zhu*, Veronica Livescu', Tyler Harrington*, Olivia Dippo`, George T. Gray III', and Kenneth S. Vecchio*^

*Department of NanoEngineering, UC San Diego, La Jolla CA, 92131

'Los Alamos National Laboratory, Materials Science and Technology Division, Los Alamos, NM 87545, ^corresponding author, kvecchio@ucsd.edu

\section{Abstract}

The influence of microstructural anisotropy on shear response of high-purity titanium was studied using the compact forced-simple-shear specimen (CFSS) loaded under quasi-static loading conditions. Postmortem characterization reveals significant difference in shear response of different directions in the same material due to material crystallographic texture anisotropy. Shear bands are narrower in specimens in which the shear zone is aligned along the direction with a strong $\{0001\}$ basal texture. Twinning was identified as an active mechanism to accommodate strains in the shear region in both orientations. This study confirms the applicability of the CFSS design for the investigation of differences in the shear response of materials as a function of process-induced crystallographic texture. A detailed, systematic approach to quantifying shear band evolution by evaluating geometrically necessary dislocations (GND) associated with crystallographic anisotropy is presented. The results show that: i) line average GND density profiles, for Ti samples that possess a uniform equiaxed-grain structure, but with strong crystallographic anisotropy, exhibit significant differences in GND density close to the shear band center; ii) GND profiles decrease steadily away from the shear band as the plastic strain diminishes, in agreement with Ashby's theory of work hardening, where the higher GND density in the through-thickness (TT) orientation, leads to a higher flow stress in the shear band compared with inplane (IP) samples; iii) the anisotropy in deformation response is derived from initial crystallographic texture of the materials, where GND density of both $<a>$ and $<c+a>$ type slip are higher adjacent to the shear band in the through-thickness sample oriented away from easy slip, corresponding to higher energy absorption; and iv) the increase in grain average GND density was determined to have strong correlation to an increase in the Euler $\Phi$ angle of the grain average orientation, indicating an increased misorientation angle evolution from $<\mathrm{a}>$ slip to $<\mathrm{c}+\mathrm{a}>$ slip.

(C) 2017. This manuscript version is made available under the Elsevier user license 
Nomenclature

$\begin{array}{cl}\beta_{i j}^{e l} & \text { Element of the elastic distortion tensor } \\ \varepsilon_{i j}^{e l} & \text { Element of the elastic strain tensor } \\ \omega_{i j} & \text { Element of the lattice rotation tensor } \\ \theta_{k} & \text { Element of the lattice rotation vector } \\ k_{i j} & \text { Element of the lattice curvature tensor } \\ \boldsymbol{\alpha} & \text { Nye dislocation tensor } \\ \delta_{i j} & \text { Element of the small deviation tensor } \\ g_{i j} & \text { Element of the lattice orientation tensor } \\ \rho_{G N D} & \text { GND density } \\ \boldsymbol{\Lambda} & \text { Lattice rotation gradient vector } \\ \boldsymbol{\xi} & \text { Active slip systems matrix } \\ \boldsymbol{\rho} & \text { Dislocation density vector }\end{array}$




\section{Introduction}

The stability of plastic deformation in a given metal or alloy, which is determined by physical, mechanical, and material/microstructural factors, has been the subject of numerous research studies [117]. Shear bands have been reported to occur in a variety of metals and alloys such as aluminum alloys, brass, steels, and titanium alloys. Moreover, shear bands have been considered failure indicators since experiments indicate that large plastic deformation involves shear localization, void nucleation, void growth, and coalescence leading to ultimate failure. It has been known for many years that shear localization is a dominant mechanism for plastic deformation in structural materials at high strains and high-strain-rate conditions and has always been considered as a precursor of catastrophic failure [1820]; however their origin, evolution, and role during plastic deformation are still not well understood nor predictably modeled.

While the shear behavior of titanium and its alloys continues to receive considerable attention owing to the significant influence shear localization has on the response of these materials in applications ranging from aerospace applications associated with crash-worthiness and foreign object damage (FOD), to military platforms required for ballistic protection, to rapid manufacturing processes such as forming and machining, the majority of the material characterization studies remain largely qualitative in nature. To overcome the limitations due to shear localization, more quantitative data on shear banding and shear location is required to develop and validate physically based constitutive models for titanium alloys that incorporate concurrent influences of temperature, strain rate, strain, and microstructural features such as texture, both crystallographic and morphological. One specific feature of interest toward understanding the plastic strain evolution in shear bands is to document the evolution of dislocation structures and their densities and relate them to variations in local work-hardening behavior.

Previous studies into shear localization in titanium alloys [17, 21-32, 33-40] have examined the propensity for shear localization at the continuum level. A subset of these studies have focused on probing the influence of crystallographic texture and how texture may play a role in promoting or mitigating the susceptibility of titanium alloys to localization [21, 22, 33, 41-43]. Texture can influence the relative orientation of grains for slip versus twinning, as well as the work-hardening capacity of individual grains relative to one another, as well as their propensity for thermal softening [33]. For example in the work of Salem and Semiatin [33], annealed CP-titanium exhibited a higher susceptibility for shear banding in the rolled plate direction compared to the transverse direction, attributed to easy slip along prismatic planes, but quantitative validation of these effects is still lacking.

One problem that has hindered the fundamental understanding of the shear banding phenomenon is the difficulty of comparing results obtained from various different types of shear loading experiments that have been developed, because shear bands have been observed to most often nucleate from geometrical defects rather than microstructural defects.

With the advent of electron backscattered diffraction (EBSD), it is now possible to probe the evolution of deformation in terms of: initial grain textures, grain crystal rotations, and quantification of deformation in terms of geometrically necessary dislocations (GNDs) that are associated with lattice curvature. This latter aspect has been discussed in more general terms by Brewer et al., [44], Field et al., [45], Ruggles et al., [46] and Calcagnotto et al., [47]. These studies demonstrate the ability and methodology to extract quantitative geometrically necessary dislocation (GND) densities from the lattice curvature as determined by EBSD within deformed samples. However, in each of these studies, the plastic 
deformation was limited to axial deformation and the extent of the deformation limited to less than $20 \%$ plastic strain.

One of the major challenges of shear banding studies, as it related to examining texture influences, is the control of the shear localization relative to the crystallographic texture that may exist in the material. The recent development of the compact-forced-simple-shear (CFSS) [48] now allows systematic variation of the formation of shear bands to be directly related to sample orientation effects, such as crystallographic or morphological grain textures. The primary objective of this paper is to employ the use of the CFSS sample to examine the effect of texture in CP-Ti and then utilize measurements of geometrically necessary dislocations (GNDs) derived from analysis of electron backscattered diffraction (EBSD) patterns to provide quantitative assessment of the shear banding behavior difference as they relate to the differences in texture. The shear response is shown to be strongly dependent on crystallographic texture. Within the shear localization regions, the plastic shear strain can easily exceed 1 , and because of the shear deformation mode, grain lattice curvatures approaching the shear band center can be very large. However, as will be demonstrated herein, with well prepared samples for EBSD analysis, it is possible to obtain EBSD patterns from very close to the shear band centerlines, determine the lattice curvatures involved as a function of the microstructure orientation, and thus quantify the extracted GND densities to differences in the crystallographic texture. Lastly, by determining differences in the GND densities, it is possible to glean details related to local strengthening differences, clarify the operative defect generation and storage mechanisms, and provide quantitative metrics for modeling of shear localization in ductile materials.

\section{Experimental}

\subsection{Materials}

Electrolytic alpha titanium, supplied by the Alta Group of Johnson Matthey Electronics, Inc., Spokane, WA was used in this study. The starting bar stock material was $38 \mathrm{~mm} \times 100 \mathrm{~mm} \times 254 \mathrm{~mm}$, and its chemical composition was verified to contain (in wt.\%) 360ppm O, 60ppm C, 10ppm N, 14ppm H, 4ppm $\mathrm{Al}, 3 \mathrm{ppm} \mathrm{V}$, $5 \mathrm{ppm} \mathrm{Fe}$, and balance titanium. The rectangular bar stock was cross-rolled from $38 \mathrm{~mm}$ to $12.5 \mathrm{~mm}$ in multiple passes at room temperature with intermediate anneals at $873 \mathrm{~K}$ for 30 minutes followed by a water quench at the steps of $50 \%$ and $75 \%$ rolling reductions. The material was recrystallized for 4 hours at $873 \mathrm{~K}$ to yield an average equiaxed grain size of $\sim 20 \mu \mathrm{m}$. Optical microscopy images and the (0002) pole figures showing the crystallographic texture of the titanium is given in Fig. 1. The rolling reduction and heat treatment schedule produced an in-plane, isotropic, basal texture in the plate with the $\{0001\}$ basal plane normals oriented nominally 20-35 degrees to the plate normal.

\subsection{Design of CFSS samples from high purity $\mathrm{Ti}$}

A large range of testing techniques and specimen geometries has been developed over the years to assess the shear behavior of metallic materials [49-54]. Some techniques are based on the fact that geometrical discontinuities in the specimen contribute to failure initiation (i.e. hat-shaped specimen, pure torsion specimen, single- or double-edged specimen [52], punch or fracture toughness specimen . Others are based on configurations in which failure stems from material behavior (i.e. torsion, multiaxial compression test, cylinder expansion test). In many cases, however, a pure shear state cannot be ensured due to bending effects induced by sample geometry with respect to the loading fixtures. 
To allow for systematic quantification of the influence of crystallographic anisotropy on the shear response of high-purity titanium, the experiments carried-out in this study were performed using the compact-forced-simple-shear (CFSS) specimen geometry presented in detail in [48]. Fig. 2 presents the geometry of the CFSS specimen used for this study. The CFSS specimen was chosen due to its adaptability to different testing platforms, which allowed testing at both low and high strain-rates. As described in [48], the size of the shear area of the sample is determined so that the maximum force generated in the shear plane does not cause plastic deformation of the adjacent shoulder region, allowing mode II in-plane shear. The specimens were approximately 5-mm diameter and $9.5-\mathrm{mm}$ long.

The major advantage of this unique sample design, as described in [48], is that its geometry allows systematic quantification of the effect of crystallographic and morphologic anisotropy on the shear response and damage evolution in addition to investigation of shear response as a function of strain rate, by allowing direct alignment of a planar shearing section in the specimen to a direction aligned to the microstructure or texture component.

\subsection{Experimental Details}

The CFSS sample geometry allows the plane of shear to be aligned with specific crystallographic directions in anisotropic materials. For this study, the CFSS samples were machined from the high-purity Ti plate with the plane of shear oriented in the through-thickness (TT) and in-plane (IP) orientations of the titanium plate, as shown in Fig. 3.

Quasi-static compression tests were performed at a nominal axial strain rate of $0.001 / \mathrm{sec}$, at an ambient temperature of $20^{\circ} \mathrm{C}$, using an electromechanical test frame. Samples were tested until a drop in load was noted as indicated by the load-displacement data.

\subsection{Metallographic analysis}

Tested specimens were sectioned through the shear zone, along the indicated direction of the plate, as shown in the 3D model in Fig. 2, and metallographically prepared using conventional methods. Sample preparation consisted of grinding on SiC paper with increasingly finer grit, followed by mechanical polishing with $0.3 \mu \mathrm{m}$ alpha alumina slurry and then a mixture of $5: 1$ by volume of $0.04 \mu \mathrm{m}$ colloidal silica and hydrogen peroxide. Electron backscattered diffraction (EBSD) and Light Optical Microscopy (LOM) were utilized to investigate the cross-sections of the specimens (highlighted in Fig. 2). In preparation for EBSD analysis, the samples were chemically polished by immersion in a solution of $6 \mathrm{~g}$ of sodium hydroxide in $50 \mathrm{ml}$ of water for 20 seconds, then another 30 seconds in a mixture of 3 drops hydrofluoric acid in $50 \mathrm{ml}$ of water.

Prior to EBSD analysis for GND calculation, a final attack polish was performed on each sample to remove the mechanically deformed surface layer imparted during previous grinding and polishing. The attack polish solution for the Ti sample was $0.05 \mu \mathrm{m}$ colloidal silica containing $15 \% \mathrm{H}_{2} \mathrm{O}_{2}$ and $7.7 \%$ Kroll's Reagent.

EBSD was conducted using a Bruker's e-Flash EBSD detector on a FEI Quanta 600 SEM at 20kV and working distance of $\sim 15 \mu \mathrm{m}$. To determine the angular resolution of the detector, a scan of a single crystal germanium sample was run using a step size of $200 \mathrm{~nm}$ and the misorientation distribution was plotted from $0-2^{\circ}$. The maximum number of occurrences of misorientation in this region was taken as 
the angular resolution of the system. In addition, step size analysis was carried out as detailed in the following section to ascertain the number of pixels ideal for our GND calculation. To ensure that the center of shear band is aligned to the top edge of the EBSD scan, the shear crack located at the end of the shear band, was aligned to the edge of the field of view, at the specified magnification of 500X, and then the sample is moved horizontally to the region of interest.

3. GND density Calculation

From a standpoint of compatibility of deformation in continuum mechanics, the Nye tensor is equal to negative the curl of lattice rotation plus elastic strain, according to Kröner [55].

$$
\alpha_{i k}=-\epsilon_{k l j} \frac{\partial \beta_{i j}^{e l}}{\partial x_{l}}=-\epsilon_{k l j} \frac{\partial \omega_{i j}}{\partial x_{l}}-\epsilon_{k l j} \frac{\partial \varepsilon_{i j}^{e l}}{\partial x_{l}}
$$

In this paper, the original Nye's relation has been adopted and the elastic strain ignored [56]; this is an appropriate assumption given the significant shear strains under investigation in the sheared regions.

$$
\alpha_{i k}=k_{k i}-\delta_{k i} k_{m m}
$$

With election backscatter diffraction (EBSD), it is now possible to extract the crystal orientation pixel by pixel from a deformed material, which leads to development of EBSD-based GND calculations from lattice orientation gradients. Since EBSD is a 2D surface technique, lattice rotation gradients in the out of plane direction are assumed to vanish $[57,58]$. Some authors proposed alternative methods involving only the experimentally determined terms [59-61], where five directly measurable terms of the Nye tensor $\alpha_{12}, \alpha_{13}, \alpha_{21}, \alpha_{23}, \alpha_{33}$ and a difference term $\alpha_{11}-\alpha_{22}$ are considered [61].

For the Nye relation to be applied in the EBSD technique, the lattice rotation tensor is approximated to the lattice orientation matrix for small deviations from the reference orientation [61].

$$
\begin{gathered}
\omega_{i j}=\mathrm{g}_{i j}-\delta_{i j} \approx \mathrm{g}_{i j} \\
\alpha_{i k}=-\epsilon_{k l j} \frac{\partial \beta_{i j}^{e l}}{\partial x_{l}} \approx-\epsilon_{k l j} \mathrm{~g}_{i j, l}
\end{gathered}
$$

For a hexagonal-close-packed crystal, the minimum misorientation or the disorientation angle could be calculated by applying 12 symmetry operators to the lattice orientation of two adjacent points of interest; the maximum misorientation in a hexagonal-close-packed crystal is $93.84^{\circ}$ [62], which could be used equivalently to determine the unique solution of misorientation that lies within the fundamental zone. Methods used for extracting the disorientation and lattice orientation gradient $g_{i j, l}$ in this paper are similar to Demir et al. [57].

To relate the geometric configuration of dislocations to dislocation density tensor, Frank's loop construction of dislocation is used, where $\hat{l}$ is the dislocation line vector, and slip direction is $b$ :

$$
\alpha_{i j}=\sum_{\mathrm{n}=1}^{N} \rho_{G N D}^{n} b_{i}^{n} \hat{l}_{j}^{n}
$$

For titanium ( $N=33), 3<a>$ screw $\langle 11 \overline{2} 0\rangle, 3<a>$ edge on basal planes $\{0001\}\langle 11 \overline{2} 0\rangle, 3<a>$ edge on prismatic planes $\{10 \overline{1} 0\}\langle 11 \overline{2} 0\rangle, 6<a>$ edge on $1^{\text {st }}$ order pyramidal planes $\{10 \overline{1} 1\}\langle 11 \overline{2} 0\rangle, 6<c+a>$ screw 
$\langle 11 \overline{2} 3\rangle$ and $12<c+a\rangle$ edge on $1^{\text {st }}$ order pyramidal planes $\{10 \overline{1} 1\}\langle 11 \overline{2} 3\rangle$ are believed to be the slip modes present according to Jones and Hutchinson [63]. In this crystal orientation based GND framework, readiness of activation for each individual type of slip due to electronic interaction of atoms will not be concerned [64, 65], since only the residual dislocation networks that has geometric consequence is being measured via an EBSD scan. Nevertheless, the adopted energy minimization scheme should be able to provide a reasonable criterion for resolving the $\langle a\rangle$ and $\langle c+a\rangle$ type dislocations $[59,66]$. A schematic diagram is shown in Fig. 4 to help visualise the slip modes in the HCP crystal structure.

In total, there are 33 types of geometric configurations of dislocations (66 if we consider the negative sign dislocations) for titanium. However, there are only nine equations that form a set of underdetermined system with 66 unknowns \{Eq. 6\}. Here, a minimum condition was made about lattice orientation in the direction normal to the surface, which is likely to result in underestimate of the dislocation density [60]. Exact solution is possible when one or two active slip systems are present [67]. $\mathrm{L}^{1}$ energy minimization scheme [59] was used here to solve for the dislocation density vector, utilizing the Optimization toolbox in Matlab (http://www.mathworks.com/).

$$
\alpha=\xi(9 \times 66) \cdot \rho(66 \times 1)=\Lambda(9 \times 1)
$$

In addition, prior to running the EBSD scan used for GND calculation, a step size analysis from 100nm to $1 \mu \mathrm{m}$ was carried out following a systematic approach of step size analysis as detailed in a paper by Jun et al. [68]. A step size of around 300nm has been chosen for this analysis following a standardised route based on how much time and resources are available to spend on computation, while maximizing accuracy of the measurement. At this step size, the lower bound of the GND density (i.e. the noise floor) was determined to be around $5.0 \times 10^{9}$ per $\mathrm{cm}^{2}$ for this set of EBSD equipment with angular resolution of about $0.4^{\circ}$, according to Wilkinson et al. [69].

\subsection{Line-averaged GND density profile (for simple shear experiment)}

As will be shown in our EBSD scans, the shear process is accompanied by a large degree of lattice rotation, the GND density associated with lattice rotation gradients would supposedly decrease away from the center of shear band. Depending on the type of anisotropy for simple shear, e.g. crystallographic, the GND density distribution would be expected to differ. More specifically, the anisotropy of the HCP crystal structure will manifest itself as differences in the GND density, in which the shear in the more favourable slip systems will impart a lower GND density as a result of lower misorientation angle evolution.

Single line profiles that are taken from a GND map as a function of distance away from the center of shear band $(y)$ readily picks up specific local variations in the GND density and neglects the overall global variation with respect to crystallographic anisotropy. This suggests a more statistically robust approach, which essentially involves taking all the line profiles possible at every y (normal to the direction of the shear band) and averaging them out in the $x$ direction (in the direction of shear band) [70]. In producing plots that present smooth data for interpretation, we could avoid doing the averaging for every y value and instead carry out the averaging at an interval of $12 \mu \mathrm{m}$. The statistical reliability of the approach is also dependent on how many available GND data points are used in the direction of shear for generation of the line average GND profiles. Clearly, the more pixels we have for each row of GND averaging, the more reliable the result. 


\section{Results and Discussion}

\subsection{Mechanical response of the materials}

The load-displacement curves for the high-purity Ti quasi-statically loaded samples are shown in Fig. 5. The load-displacement responses of the high-purity Ti tested in shear differ substantially consistent with the large differences in the yield stresses of high-purity $\mathrm{Ti}$ [71] previously documented in the literature between in-plane and through-thickness orientations. The high-purity Ti displays a significant difference in its shear response between the TT and IP directions consistent with the strong basal texture of the material [72], making the TT direction the higher flow stress direction. Shear loading in both the TT and IP directions for the high-purity Ti displayed significant strain hardening, more than a factor of 2 increase in load carrying capacity after yield, with the hardening rate higher in the TT sample, as indicated in Fig. 5. Recorded peak loads in these specimens during quasi-static tests for the high-purity IP and TT directions were $520 \mathrm{~N}$ and $850 \mathrm{~N}$, respectively. In addition, the IP direction specimen recorded a 0.8-mm displacement at load drop, compared to $0.55-\mathrm{mm}$ for the TT direction. The documented slip modes in Ti [73] in order of ease of operation, are: $\{10 \overline{1} 0\}$ prism, $\{10 \overline{1} 1\}$ first-order pyramidal, and $\{0001\}$ basal slip, respectively, with the $\langle 11 \overline{2} 0\rangle$ slip burgers directions. The Schmid factors applied to these slip systems, upon altering the macroscopic loading direction relative to the starting texture, have a large effect on: a) the stress levels necessary to activate slip, b) the trade-offs between slip and twinning deformation modes to accommodate the applied stresses, and c) the overall strain-hardening rate in $\mathrm{Ti}$ due to the number and specific slip and twinning systems activated. Compressive shear loading in the TT plate direction results in relatively unfavorable Schmid factors for easy activation of prism, pyramidal, and basal slip. Accordingly, deformation in this loading orientation will result in higher flow stress levels and favor deformation twinning to accommodate c-axis contraction in $\mathrm{Ti}$, consistent with the maximum load values in Fig. 5 and metallographic observations. Conversely, compressive loading applied to the IP samples will have Schmid factors favoring activation of prism, pyramidal, and basal slip, thereby requiring lower flow stresses to initiate and sustain plastic flow. In addition, IP loading of the high-purity Ti will result in a reduced propensity for deformation twinning due to the reduced necessity to accommodate c-axis contraction and/or expansion.

The shear strain $(\bar{\gamma})$ of the CFSS high-purity samples can be estimated by dividing the average shear displacement $(\overline{\mathrm{u}})$ with the shear band's width (w). From the optical micrographs in Fig. 6 , it can be seen that there are errors associated with making estimates of both the shear-band widths and average shear displacement, yielding errors ranging from $5 \%$ to $10 \%$ for the computed shear strain. Table 1 summarizes measurements and calculations of shear strains for the IP and TT Ti shear samples. As shown in Table 1, CFSS samples undergo severe plastic shear deformation, where the highest shear strain approaches 1.2. Nevertheless, the following sections will show that reasonably good EBSD scans for GND analysis could still be obtained.

Table 1. Estimate of shear strain $(\bar{\gamma})$ and error $\left(\sigma_{\bar{\gamma}}\right)$ of the CFSS samples of high -titanium from average shear displacement $(\overline{\mathbf{u}})$ and shear band's width $(\mathrm{w})$.

\begin{tabular}{cc|cccc}
\multicolumn{1}{c}{} & $\mathrm{w}(\mu \mathrm{m})$ & $\overline{\mathrm{u}}(\mu \mathrm{m})$ & $\bar{\gamma}$ & $\sigma_{\bar{\gamma}}$ \\
\hline High-purity Ti & IP & 314 & 390 & 1.24 & 0.09 \\
& TT & 428 & 312 & 0.73 & 0.04
\end{tabular}




\subsection{Shear Damage Evolution - Post Mortem Optical Microscopy}

Light optical micrographs of the sheared cross-sections of the CFSS samples of the high-purity $\mathrm{Ti}$ following quasi-static testing are shown in Fig. 6. As presented in Fig. 6, the high-purity Ti in the IP direction displayed substantial diffuse plasticity and significant displacement of the sheared volume in line with the modestly large overall sample displacement. The TT direction for the high-purity Ti, which is the "hard" crystallographic direction at approximately 20-35 degrees off the c-axis, also displayed extensive plasticity in the shearing region. In addition, at the point of the load drop when testing was stopped, a slightly increased number of voids and interconnected cracking is seen within the shearing zone.

\subsection{GND Density Calculation-Crystallographic anisotropy}

The observation here of differences in the extent of the plastic shear zone between the IP and TT sample orientations must be due to differences in the evolution of geometrically necessary dislocations (GND) versus statistically stored dislocations (SSD). Shear in orientations aligned parallel to favorable slip systems (IP samples) will result in fewer geometrically necessary dislocations (related to the lower misorientation angle evolution in grains), while less favorably oriented slip systems (TT samples) will necessitate the evolution of larger numbers of geometrically necessary dislocations (i.e. more grain curvature). By contrast, differences in flow stress levels are responsible for the differences in the extent of the plastic zone away from the shear band center. A higher initial flow stress results in attaining the saturation stress more rapidly within the shear band. Once the saturation stress is achieved within the shear band and intense localization begins, no additional plasticity occurs away from the shear band center, as all flow becomes localized in the saturated stress region. As such, one would expect the statistically stored dislocation density to extend over a wider region adjacent to the shear band in lower strength materials, while the density of geometrically necessary dislocations will scale with the relative misorientation of the shear plane with respect to the easy slip system orientations. In the following section, GND density evolution is examined with specific focus on the significant difference in mechanical responses due to initial texture of the material.

As previously reported by many other studies conducted on titanium alloys, initial crystallographic texture of Ti-based alloys leads to different deformation response for the materials, i.e. crystallographic anisotropy [36, 74-76]. For a quantitative analysis of the effect of crystallographic anisotropy in terms of geometrically necessary dislocations, the line average GND density profiles and grain average GND density plotted in orientation space have been produced in Fig. 7 and Fig. 8 to glean information about GND evolution starting from the center of the shear band to adjacent less deformed regions and the influence of crystal orientation of a grain on GND generation.

The cross section of the in-plane (IP) pure titanium sample that was machined in the rolling direction displays an apparent $\{0001\}$ basal texture, see Fig. 7. This type of basal texture pre-disposes the easy $<a>$ type slip mode, i.e. 'soft mode' to deformation, which results in lower flow stresses during shearing. However, the cross section of the through-thickness (TT) sample cut transverse to the rolling direction, as shown in Fig. 7, shows some non-basal texture in $\{10 \overline{1} 0\}$ and $\{11 \overline{2} 0\}$. Consequently, the orientations of the crystals for TT sample lead to more $\langle c+a\rangle$ type slip to be activated during plastic deformation, i.e. 'hard mode' [74]. Notice that deformation twining is also present in both of the pure Ti samples because twinning is thought to be an active mechanism to accommodate strain once large enough stresses are operative on the twinning system [77]. 
Fig. 7 shows an area selected for the GND density calculation for each of the IP and TT samples. It can be seen that, for example, a region of high GND density is present at the center of the selected area in Fig. 7 that corresponds with the highly distorted grain in the EBSD image. Similarly, for grains that appear to have no change or very little change in their orientation, GND "cold pockets" (areas of low dislocation density) are found. Moreover, some GND "hotspots" are clearly seen, most commonly at triple junctions. This is consistent with the added compatibility constraints at grain triple points. Additionally, some dislocation cell structures for $\langle c+a\rangle$ type dislocations are also visible in Fig. 7 .

To demonstrate the effect of crystallographic orientation on GND density, a new method is presented; see Fig. 8, in which grain average GND density is plotted against the grain average orientation. The analysis is based on the assumption that shear related grain orientation changes associated with the generation of GND will not significantly alter the grain average orientation, which is reasonable based on the EBSD images in Fig. 8. It is found that the grain average orientation for the IP direction is more random than for the TT direction. The IP direction in Fig. 8 consists of: (a) grains oriented mainly with the easy slip plane orientation to the shear direction (blue) that favours the <a>type slip, (b) a few 'hard' mode grains (red) favouring the $\langle\mathrm{c}+\mathrm{a}>$ type slip, and (c) several grains in the transition state from 'soft' to 'hard' mode (green). The dependence of the total GND density in terms of the three Euler angles $\left(\varphi_{1}, \Phi, \varphi_{2}\right)$ for the IP direction is plotted in Fig. 8. It is clear how rapidly the order of magnitude of total GND density increases linearly as the Euler angle $\Phi$ increases, whereas the dependence on the Euler angles, $\varphi_{1}$ and $\varphi_{2}$ is relatively weak. For the same relative amount of increase in GND density, the change in $\Phi$ is around $30^{\circ}$, but the change in $\varphi_{1}$ or $\varphi_{2}$ is at least 100 . In other words, the rate of change of total GND density with respect to $\Phi$ angle is almost three times greater than the other two Euler angles $\varphi_{1}$ and $\varphi_{2}$. Therefore, it can be concluded that the change of GND density variation due to crystallographic texture is mainly derived from the difference in the Euler angle $\Phi$ for HCP structured titanium. This is in agreement with expectation since at low $\Phi$ angles, the favorable $<a>$ type slip will produce lower misorientation angle evolution, whereas <c+a> type slip operated at higher $\Phi$ angles requires a larger amount of GND to maintain the compatibility constraints, as for example, in the TT direction.

Fig. 9 shows the statistical reliability of the line average GND density profile approach. The distortion of the crystals is not significant enough to result in degraded patterns near the shear band. A large number of fragments of grains near the shear bands were clearly indexed via EBSD. Near the centerline of the shear band, at $y=0$, the number of GND pixels decreases by an order of magnitude because of pattern degradation associated with severe plastic shear strain. As the number of pixels is generally lower in the TT sample near the shear band compared with the IP sample, it is expected that a higher GND density exists near the shear band for the TT sample. Moreover, it can be seen that the number of GND data points for TT and IP samples converge at a distance further away from the shear band, which implies that the discrepancy in GND would be reduced moving into the less deformed region.

Fig. 10(a) shows the direct comparison of the line average GND density profiles for the TT and IP samples. Near the center of the shear band, the TT sample has a much higher total GND density than the IP sample, consistent with the results presented in Fig. 9. This agrees well with Britton et al.'s analogous study on the effect of crystallographic texture in Ti to the GND distribution near indents [59], in which the indentation along the basal direction results in a lower GND distribution near the indent due to the easier <a>-type slip. In addition, differences in the line average total GND density profiles decreases as expected near the less deformed region as plastic shear strain diminishes away from shear band's center. These features are also observed in the separate <a> [Fig. 10(b)] and <c+a> [Fig. 10(c)] type GND density profiles, which indicates that the TT sample's lattice rotation during simple shear 
requires more $\langle a>$ and $\langle c+a>$ type slip than for the IP sample. It has also been found that the net resultant $<a>$ type GND density is, in general, higher than <c+a> type GND density in both samples, independent of the initial texture. The rate at which the line average GND density for both $<a>$ and $<c+a>$ type slip decreases is faster for the TT sample, which implies that more GNDs are required for the TT sample to accommodate the lattice rotations closer to the shear band, consistent with what has been shown in Fig. 8 for the orientation dependence of GND. All these observations suggest that shearing of the TT sample requires a higher flow stress than does the IP sample, consistent with Ashby's theory [78]. The fact that the GND density is higher for the TT sample also implies that the energy absorbed during simple shear for the TT sample is higher than that needed in the IP sample, as more mechanical work is required to generate, at least more geometrically necessary, dislocations.

\section{Conclusions}

The influence of crystallographic anisotropy on the shear response of high-purity polycrystalline titanium was studied using the compact forced-simple-shear specimen (CFSS) loaded at quasi-static strain rate conditions. In this paper, a systematic method is presented, based on the emerging EBSD technique, to quantitatively analyse the number density of geometrically necessary dislocations associated with simple shear of CFSS samples for high-purity titanium. These findings provide insights into the shear responses of crystallographically anisotropic engineering materials, and illustrate how the effect of crystallographic anisotropy determines the deformation response of high-purity, textured titanium. Our observations are consistent with previous published research on the shear response of materials as well as the theories of dislocation generation and storage during plastic deformation. More specifically, it was observed for shear loading in high-purity titanium that:

- The shear response is strongly dependent on crystallographic texture.

- When slip is restricted in titanium due to texture, the material displays an inhomogeneous deformation response, even at low to moderate plastic strains, leading to strain localization upon forced shear loading. The strong basal texture in the high-purity $\mathrm{Ti}$ is reflected in approximately $50 \%$ higher yield strength and reduced displacement prior to localization for quasi-static shear loading in the TT direction compared to the IP direction.

- The number of available GND data points decreases nearer to the center of a shear band as a result of pattern degradation associated with the increasing plastic strain in the interior of a shear band.

- Away from the shear band, the line average GND density, in general, for all types of dislocations, was noted to drop, consistent with Ashby's theory on work hardening of materials.

- In the high-purity titanium samples, the damage evolution for the in-plane and through thickness samples was seen to be generally similar, whereas the lattice rotation associated with shear was found to be significantly different near the shear band, with the through thickness sample having a much higher overall GND density near the center of shear band for both <a> and $<\mathrm{c}+\mathrm{a}>$ type slip.

- GND density was determined to vary linearly with the Euler angle $\Phi$ of the grain average orientation.

- Using a $L^{1}$ dislocation energy minimization scheme, <a> type slip modes were found to be in general more favoured than $\langle c+a\rangle$ type slip, regardless of the initial texture of the sample.

- It is quantitatively confirmed that the deformation response of the high-purity Ti throughthickness samples will display a 'hard mode' from an energy absorption perspective by considering the GND generated for both $<a>$ and $<c+a>$ type slip to accommodate the lattice rotation, in agreement with experimental results. 


\section{Acknowledgements}

Los Alamos National Laboratory (LANL) is operated by Los Alamos National Security, LLC, for the National Nuclear Security Administration of the U.S. Department of Energy under contract DE-AC5206NA25396. The LANL work was partially sponsored by the Joint DoD/DOE Munitions Technology Development Program. Chaoyi Zhu would like to acknowledge insightful discussions with Dr. Ben Britton and Dr. Jun Jiang from Imperial College London.

\section{References}

[1] L.W. Meyer, F. Pursche, Experimental methods, in: B. Dodd, Y. Bai (Eds.), Adiabatic Shear Localization: Frontiers and Advances, Elsevier, Amsterdam, The Netherlands, 2012, pp. 21-110.

[2] J.S. Rinehart, J. Pearson, Behavior of Metals Under Impulsive Loads, American Society for Metals, Cleveland, Ohio, 1954.

[3] J.R. Klepaczko, H.V. Nguyen, W.K. Nowacki, Quasi-static and dynamic shearing of sheet metals, European Journal of Mechanics-A/Solids 18(2) (1999) 271-289.

[4] L. Anand, S.R. Kalidindi, The process of shear band formation in plane strain compression of fcc metals: effects of crystallographic texture, Mechanics of Materials 17(2-3) (1994) 223-243.

[5] N. Chung, J. Embury, J. Evensen, R. Hoagland, C. Sargent, Unstable shear failure in a 7075 aluminum alloy, Acta Metall Mater 25(4) (1977) 377-381.

[6] A.S. Argon, Stability of plastic deformation, in: R. E. Reed-Hill (Ed.), The In-homogeneity of Plastic Deformation, American Society for Metals, Metals Park, Ohio, 1973, pp. 161-190.

[7] Y. Xu, J. Zhang, Y. Bai, M.A. Meyers, Shear localization in dynamic deformation: microstructural evolution, Metallurgical and materials transactions A 39(4) (2008) 811-843.

[8] Q. Xue, X. Liao, Y. Zhu, G.T. Gray III, Formation mechanisms of nanostructures in stainless steel during high-strain-rate severe plastic deformation, Materials Science and Engineering: A 410 (2005) 252-256.

[9] M. Meyers, Plasticity: Adiabatic shear localization, in: K. H. J. Buschow, R. W. Cahn, M. C. Flemings, B. Illschner, E. J. Kramer, S. Mahajan (Eds.) Encyclopedia of Materials: Science and Technology, Elsevier, 2001, pp. 7093-7103.

[10] L.W. Meyer, E. Staskewitsch, A. Burblies, Adiabatic shear failure under biaxial dynamic compression/shear loading, Mechanics of Materials 17(2) (1994) 203-214.

[11] Q. Xue, J.F. Bingert, B.L. Henrie, G.T. Gray III, EBSD characterization of dynamic shear band regions in pre-shocked and as-received 304 stainless steels, Materials Science and Engineering: A 473(1) (2008) 279-289.

[12] Q. Xue, E.K. Cerreta, G.T. Gray III, Microstructural characteristics of post-shear localization in coldrolled 316L stainless steel, Acta Mater 55(2) (2007) 691-704.

[13] Q. Xue, G.T. Gray III, Development of adiabatic shear bands in annealed 316L stainless steel: Part II. TEM studies of the evolution of microstructure during deformation localization, Metallurgical and Materials Transactions A 37(8) (2006) 2447-2458. 
[14] Q. Xue, G.T. Gray III, Development of adiabatic shear bands in annealed 316L stainless steel: Part I. Correlation between evolving microstructure and mechanical behavior, Metallurgical and Materials Transactions A 37(8) (2006) 2435-2446.

[15] Q. Xue, G.T. Gray III, B. Henrie, S. Maloy, S. Chen, Influence of shock prestraining on the formation of shear localization in 304 stainless steel, Metallurgical and Materials Transactions A 36(6) (2005) 1471-1486.

[16] Y. Zhang, G. Liu, X. Han, Analysis of strain localization for ductile materials with effect of void growth, International Journal of Mechanical Sciences 46(7) (2004) 1021-1034.

[17] Q. Xue, M.A. Meyers, V.F. Nesterenko, Self-organization of shear bands in titanium and Ti-6Al-4V alloy, Acta Mater 50(3) (2002) 575-596.

[18] D. Rittel, S. Osovski, Dynamic failure by adiabatic shear banding, International Journal of Fracture 162(1-2) (2010) 177-185.

[19] D. Rittel, Z. Wang, Thermo-mechanical aspects of adiabatic shear failure of AM50 and Ti6Al4V alloys, Mechanics of materials 40(8) (2008) 629-635.

[20] D. Rittel, Z. Wang, M. Merzer, Some experiments on adiabatic shear failure, Journal de Physique IV (Proceedings), EDP sciences, 2006, pp. 835-838.

[21] S.E. Schoenfeld, B. Kad, Texture effects on shear response in Ti6Al4V plates, Int. J. Plast. 18 (2002) 461-486.

[22] B.K. Kad, S.E. Schoenfeld, M.S. Burkins, Through thickness dynamic impact response in textured Ti6Al4V plates, Mater. Sci. Engng A 322 (2002) 241-251.

[23] J.R. Klepaczko, Behavior of Ti-6Al-4V alloy at high strain rates, shear testing up to $6 \times 10^{4} \mathrm{~s}^{-1}$ and failure criterion, J. Phys. IV France Pr. 9 (DYMAT 2000) 10 (2000) 191-196.

[24] M.G. da Silva, K.T. Ramesh, The rate dependent deformation and localization of fully dense and porous Ti-6Al-4V, Mater. Sci. Engng A 232 (1997) 11-22.

[25] S.V. Kailas, Y.V.R.K. Prasad, S.K. Biswas, Flow instabilities and fracture in Ti-6Al-4V deformed in compression at 298K to 673K, Metall. mater. Trans. A 25 (1994) 2173-2180.

[26] Y.L. Bai, Q. Xue, Y.B. Xu, L. Shen, Characteristics and microstructure in the evolution of shear localization in Ti-6Al-4V alloy, Mech. Mater. 17 (1994) 155-164.

[27] A.S. Khan, S. Yu, H. Liu, Deformation induced anisotropic responses of Ti-6Al-4V alloy Part II: A strain rate and temperature dependent anisotropic yield criterion, International Journal of Plasticity 38 (2012) 14-26.

[28] A.S. Khan, S. Yu, Deformation induced anisotropic responses of Ti-6Al-4V alloy. Part I: Experiments, International Journal of Plasticity 38 (2012) 1-13.

[29] A.S. Khan, R. Kazmi, B. Farrokh, Multiaxial and non-proportional loading responses, anisotropy and modeling of Ti-6Al-4V titanium alloy over wide ranges of strain rates and temperatures, International Journal of Plasticity 23(6) (2007) 931-950.

[30] A.S. Khan, Y.S. Suh, R. Kazmi, Quasi-static and dynamic loading responses and constitutive modeling of titanium alloys, International Journal of Plasticity 20(12) (2004) 2233-2248.

[31] G. Ye, S. Xue, M. Jiang, X. Tong, L. Dai, Modeling periodic adiabatic shear band evolution during high speed machining Ti-6Al-4V alloy, International journal of plasticity 40 (2013) 39-55. 
[32] X. Li, G. Guo, J. Xiao, N. Song, D. Li, Constitutive modeling and the effects of strain-rate and temperature on the formability of Ti-6Al-4V alloy sheet, Materials \& Design 55 (2014) 325-334.

[33] A. Salem, S. Semiatin, Anisotropy of the hot plastic deformation of Ti-6Al-4V single-colony samples, Materials Science and Engineering: A 508(1) (2009) 114-120.

[34] B. Wang, Y. Yang, Z. Chen, Y. Zeng, Adiabatic shear bands in $\alpha$-titanium tube under external explosive loading, Journal of materials science 42(19) (2007) 8101-8105.

[35] D.R. Chichili, K. Ramesh, K.J. Hemker, Adiabatic shear localization in $\alpha$-titanium: experiments, modeling and microstructural evolution, Journal of the Mechanics and Physics of Solids 52(8) (2004) 1889-1909.

[36] S.V. Kailas, Y. Prasad, S. Biswas, Influence of initial texture on the microstructural instabilities during compression of commercial $\alpha$-titanium at $25 \mathrm{C}$ to $400 \mathrm{C}$, Metallurgical and Materials Transactions A 25(7) (1994) 1425-1434.

[37] Z. Wyatt, W. Joost, D. Zhu, S. Ankem, Deformation mechanisms and kinetics of time-dependent twinning in an $\alpha$-titanium alloy, International Journal of Plasticity 39 (2012) 119-131.

[38] B. Morrow, R. Lebensohn, C. Trujillo, D. Martinez, F. Addessio, C. Bronkhorst, T. Lookman, E. Cerreta, Characterization and modeling of mechanical behavior of single crystal titanium deformed by split-Hopkinson pressure bar, International Journal of Plasticity 82 (2016) 225-240.

[39] M.E. Nixon, O. Cazacu, R.A. Lebensohn, Anisotropic response of high-purity $\alpha$-titanium: Experimental characterization and constitutive modeling, International Journal of Plasticity 26(4) (2010) 516-532.

[40] Y. Yang, L. Wang, C. Zambaldi, P. Eisenlohr, R. Barabash, W. Liu, M. Stoudt, M. Crimp, T. Bieler, Characterization and modeling of heterogeneous deformation in commercial purity titanium, Jom 63(9) (2011) 66-73.

[41] R.L. Woodward, Metallographic features associated with the penetration of titanium alloy targets, Metall. Trans. A 10 (1979) 569-573.

[42] Z. Zhang, T.-S. Jun, T.B. Britton, F.P. Dunne, Intrinsic anisotropy of strain rate sensitivity in single crystal alpha titanium, Acta Materialia 118 (2016) 317-330.

[43] Z. Zhang, D.E. Eakins, F.P. Dunne, On the formation of adiabatic shear bands in textured HCP polycrystals, International Journal of Plasticity 79 (2016) 196-216.

[44] L. N. Brewer, D.P. Field, C.C. Merriman, in: A. J. Schwartz et al (Ed.), Electron Backscatter Diffraction in Materials Science2009, pp. 251-262.

[45] D.P. Field, C.C. Merriman, N. Allain-Bonasso, F. Wagner, Quantification of dislocation structure heterogeneity in deformed polycrystals by EBSD, Modelling and Simulation in Materials Science and Engineering 20(2) (2012) 024007.

[46] T. Ruggles, D. Fullwood, J. Kysar, Resolving geometrically necessary dislocation density onto individual dislocation types using EBSD-based continuum dislocation microscopy, International Journal of Plasticity 76 (2016) 231-243.

[47] M. Calcagnotto, D. Ponge, E. Demir, D. Raabe, Orientation gradients and geometrically necessary dislocations in ultrafine grained dual-phase steels studied by 2D and 3D EBSD, Materials Science and Engineering: A 527(10) (2010) 2738-2746. 
[48] G.T. Gray III, K.S. Vecchio, V. Livescu, Compact forced simple-shear sample for studying shear localization in materials, Acta Mater 103 (2016) 12-22.

[49] R. Tham, Experimental method for the evaluation of the susceptibility of materials to shear band formation, EPJ Web of Conferences, EDP Sciences, 2012, p. 01016.

[50] J. Peirs, P. Verleysen, J. Degrieck, Novel technique for static and dynamic shear testing of Ti6Al4V sheet, Experimental mechanics 52(7) (2012) 729-741.

[51] D. Rittel, S. Lee, G. Ravichandran, A shear-compression specimen for large strain testing, Experimental Mechanics 42(1) (2002) 58-64.

[52] J.F. Kalthoff, Modes of dynamic shear failure in solids, International Journal of Fracture 101(1-2) (2000) 1-31.

[53] M. Meyers, G. Subhash, B. Kad, L. Prasad, Evolution of microstructure and shear-band formation in $\alpha$-hcp titanium, Mechanics of materials 17(2) (1994) 175-193.

[54] J. Klepaczko, Some results and new experimental technique in studies of adiabatic shear bands, Archives of Mechanics 46(1-2) (1994) 201-229.

[55] E. Kroner, Continuum Theory of Dislocations and Self-Stresses, Springer 1958.

[56] J.F. Nye, Some geometrical relations in dislocated crystals, Acta Metall Mater 1(2) (1953) 153-162.

[57] E. Demir, D. Raabe, N. Zaafarani, S. Zaefferer, Investigation of the indentation size effect through the measurement of the geometrically necessary dislocations beneath small indents of different depths using EBSD tomography, Acta Mater 57(2) (2009) 559-569.

[58] S. Sun, B. Adams, W. King, Observations of lattice curvature near the interface of a deformed aluminium bicrystal, Philosophical Magazine A 80(1) (2000) 9-25.

[59] T.B. Britton, H. Liang, F.P.E. Dunne, A.J. Wilkinson, The effect of crystal orientation on the indentation response of commercially pure titanium: experiments and simulations, $P$ R Soc A 466(2115) (2010) 695-719.

[60] T. Ruggles, D. Fullwood, Estimations of bulk geometrically necessary dislocation density using high resolution EBSD, Ultramicroscopy 133 (2013) 8-15.

[61] W. Pantleon, Resolving the geometrically necessary dislocation content by conventional electron backscattering diffraction, Scripta Mater 58(11) (2008) 994-997.

[62] A. Heinz, P. Neumann, Representation of orientation and disorientation data for cubic, hexagonal, tetragonal and orthorhombic crystals, Acta Crystallographica Section A: Foundations of Crystallography 47(6) (1991) 780-789.

[63] I. Jones, W. Hutchinson, Stress-state dependence of slip in titanium-6AI-4V and other HCP metals, Acta Metall Mater 29(6) (1981) 951-968.

[64] M. Ghazisaeidi, D. Trinkle, Core structure of a screw dislocation in Ti from density functional theory and classical potentials, Acta Materialia 60(3) (2012) 1287-1292.

[65] N. Tarrat, M. Benoit, D. Caillard, L. Ventelon, N. Combe, J. Morillo, Screw dislocation in hcp Ti: DFT dislocation excess energies and metastable core structures, Modelling and Simulation in Materials Science and Engineering 22(5) (2014) 055016. 
[66] F. Dunne, R. Kiwanuka, A. Wilkinson, Crystal plasticity analysis of micro-deformation, lattice rotation and geometrically necessary dislocation density, Proc. R. Soc. A, The Royal Society, 2012, pp. 2509-2531.

[67] J. Kysar, Y. Saito, M. Oztop, D. Lee, W. Huh, Experimental lower bounds on geometrically necessary dislocation density, International Journal of Plasticity 26(8) (2010) 1097-1123.

[68] J. Jiang, T.B. Britton, A.J. Wilkinson, Measurement of geometrically necessary dislocation density with high resolution electron backscatter diffraction: Effects of detector binning and step size, Ultramicroscopy 125 (2013) 1-9.

[69] A.J. Wilkinson, D. Randman, Determination of elastic strain fields and geometrically necessary dislocation distributions near nanoindents using electron back scatter diffraction, Philosophical Magazine 90(9) (2010) 1159-1177.

[70] C. Zhu, T. Harrington, V. Livescu, G.T. Gray lii, K.S. Vecchio, Determination of geometrically necessary dislocations in large shear strain localization in aluminum, Acta Mater 118 (2016) 383394.

[71] G. Gray III, Influence of strain rate and temperature on the structure. Property behavior of highpurity titanium, Le Journal de Physique IV 7(C3) (1997) C3-423-C3-428.

[72] J. Millett, G. Whiteman, N. Bourne, G. Gray III, The role of anisotropy in the response of the titanium alloy Ti-6Al-4V to shock loading, Journal of Applied Physics 104(7) (2008) 073531.

[73] H. Padilla, J. Lambros, A. Beaudoin, I. Robertson, Relating inhomogeneous deformation to local texture in zirconium through grain-scale digital image correlation strain mapping experiments, International Journal of Solids and Structures 49(1) (2012) 18-31.

[74] B.K. Kad, S.E. Schoenfeld, M.S. Burkins, Through thickness dynamic impact response in textured Ti-6Al-4V plates, Materials Science and Engineering: A 322(1) (2002) 241-251.

[75] S.E. Schoenfeld, B. Kad, Texture effects on shear response in Ti-6Al-4V plates, International Journal of Plasticity 18(4) (2002) 461-486.

[76] J.S. Weaver, M.W. Priddy, D.L. McDowell, S.R. Kalidindi, On capturing the grain-scale elastic and plastic anisotropy of alpha-Ti with spherical nanoindentation and electron back-scattered diffraction, Acta Materialia 117 (2016) 23-34.

[77] T.B. Britton, F. Dunne, A. Wilkinson, On the mechanistic basis of deformation at the microscale in hexagonal close-packed metals, Proceedings of the Royal Society of London A: Mathematical, Physical and Engineering Sciences, The Royal Society, 2015, p. 20140881.

[78] M. Ashby, The deformation of plastically non-homogeneous materials, Philos Mag 21(170) (1970) 399-424.

[79] K. Momma, F. Izumi, VESTA: a three-dimensional visualization system for electronic and structural analysis, Journal of Applied Crystallography 41(3) (2008) 653-658.

[80] F. Bachmann, R. Hielscher, H. Schaeben, Texture analysis with MTEX-free and open source software toolbox, Solid State Phenomena 160 (2010) 63-68. 
Figure Captions:

Fig. 1. (a) Optical metallography of the high-purity Alta-Ti, and (b) [0002] alpha-phase pole figures for the Alta high-purity Ti showing the strong basal texture in the plane of the plate.

Fig. 2. Schematic of Compact Simple-Shear Sample (CFSS)

Fig. 3. Orientation of CFSS samples sectioned for shear studies relative to plate and bar stock

Fig. 4. Different slip modes present in HCP titanium $<a>$ type $<11 \overline{2} 0\rangle$ slip on basal plane $\{0001\}$, prismatic plane $\{10 \overline{1} 0\}$, pyramidal plane $\{10 \overline{1} 1\}$ and $\langle c+a>$ type $\langle 11 \overline{2} 3\rangle$ slip on pyramidal plane $\{10 \overline{1} 1\}$, plotted with VESTA [79] where the black box is one unit cell of the corresponding crystal structure.

Fig. 5. Load (N) versus displacement for the Alta (high-purity) Ti under quasi-static loading condition.

Fig. 6. Light optical images of quasi-statically tested CFSS samples showing shear band regions.

Fig. 7. High-purity titanium with shear zone aligned in in-plane and through-thickness direction. Rows: $1^{\text {st }}$, in-plane (IP) sample; $2^{\text {nd }}$, through-thickness (TT) sample. Column: $1^{\text {st }}$, EBSD image with selected example region showing the GND density map plotted with MTEX (Scale bar: $100 \mu \mathrm{m}$ ) [80]; $2^{\text {nd }}$, Total GND density map of the selected region (unit: $1 / \mathrm{cm}^{2}$ ) with $20 \mu \mathrm{m}$ scale bar; $3^{\text {rd }}$, GND density map of <a> type dislocations (unit: $1 / \mathrm{cm}^{2}$ ) with $20 \mu \mathrm{m}$ scale bar; $4^{\text {th }}$, GND density map of $\langle\mathrm{c}+\mathrm{a}>$ type dislocations (unit: $1 / \mathrm{cm}^{2}$ ) with $20 \mu \mathrm{m}$ scale bar.

Fig. 8. Effect of grain average orientation on grain average GND density (log10 scale, unit: $\left.1 / \mathrm{cm}^{2}\right)$. Row: $1^{\text {st }}$, grain average GND plotted with respect to three Euler angles $\left(\varphi_{1}, \Phi, \varphi_{2}\right)$ defining the mean orientation of the grain for in-plane high-purity Ti sample; $2^{\text {nd }}$, grain average GND plotted with respect to three Euler angles $\left(\varphi_{1}, \Phi, \varphi_{2}\right)$ defining the mean orientation of the grain for through-thickness high-purity Ti sample. Column: $1^{\text {st }}, 3 \mathrm{D}$ scatter plots of the grain average GND density in the Euler angles' space; plots of grain average GND density with respect to Euler angles $\left(2^{\text {nd }}\right) \varphi_{1}\left(3^{\text {rd }}\right) \Phi$ and $\left(4^{\text {th }}\right) \varphi_{2}$ for in-plane (top row) and through-thickness (bottom row) high-purity titanium samples, respectively.

Fig. 9. Number of available GND data points for line average GND density analysis in the $y$ direction: blue circle is for the through-thickness high-purity Ti sample; green circle is for in-plane high-purity Ti sample.

Fig. 10. Comparison of line average GND density as a function of distance away from the center of shear band for (left) total GND density, (middle) <a> type GND density and (right) $<c+a>$ type GND density for in-plane and through-thickness high-purity Ti samples (log10 scale, unit: $1 / \mathrm{cm}^{2}$ ). 

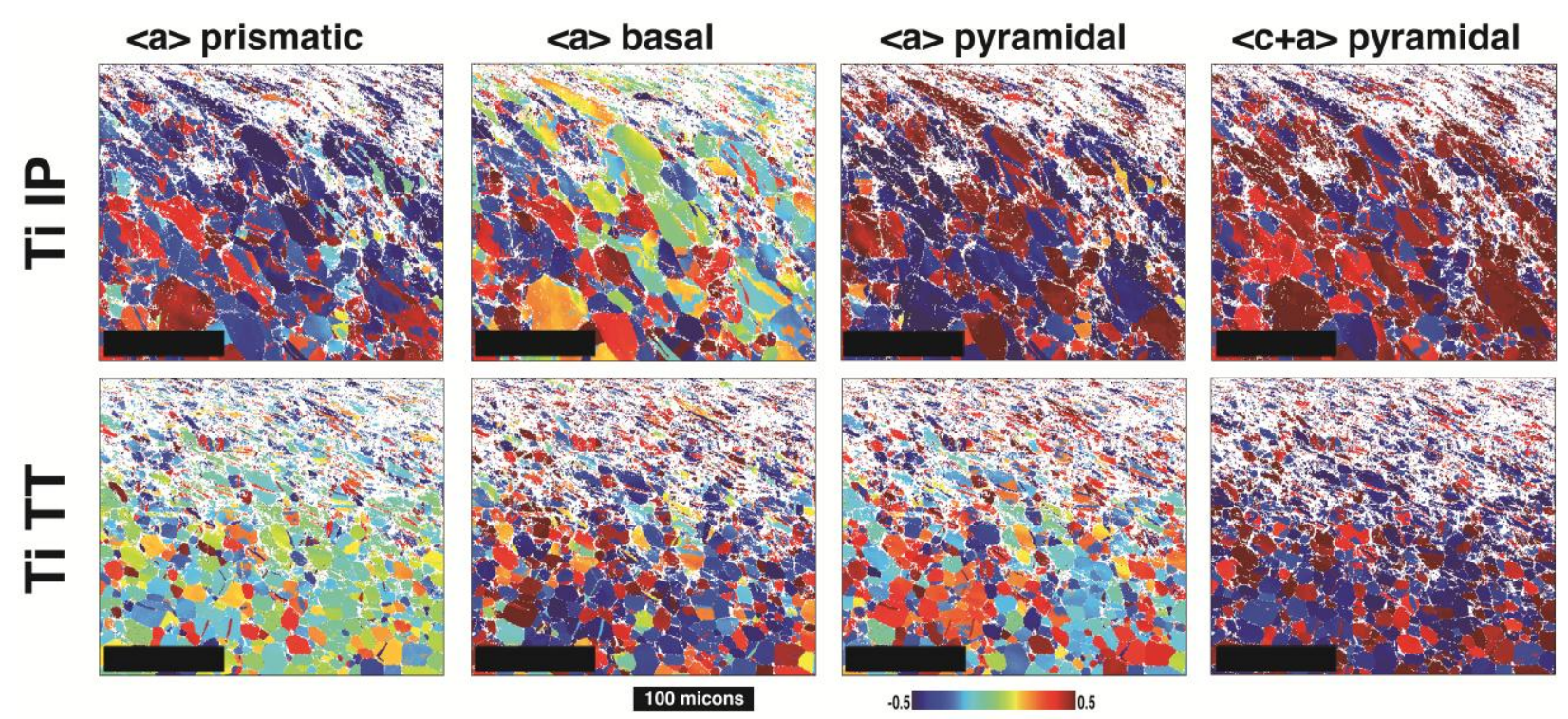

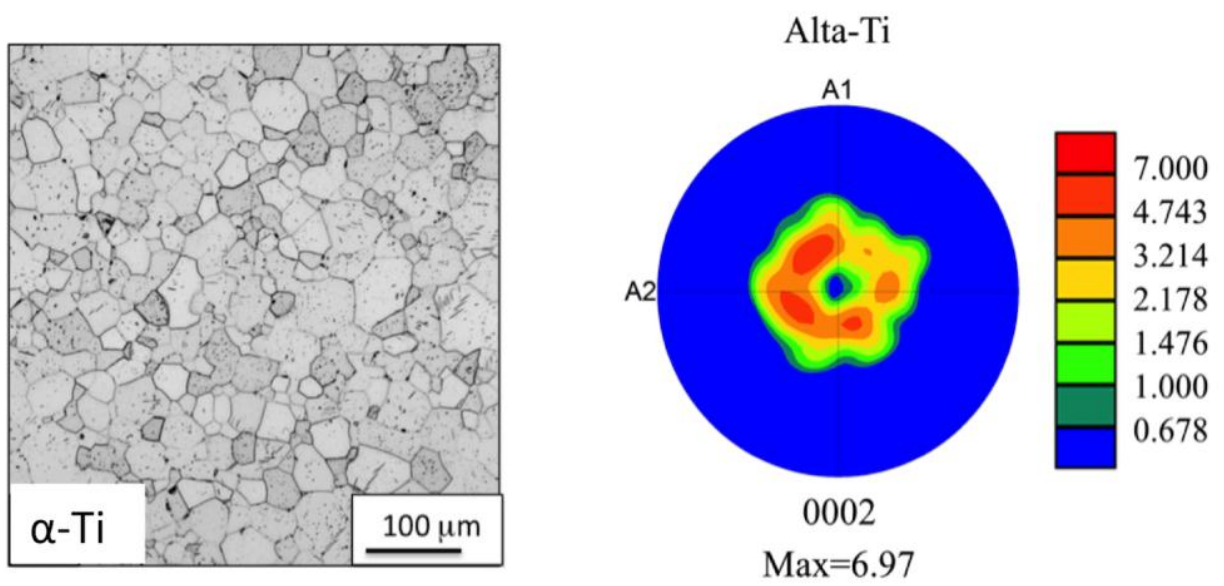

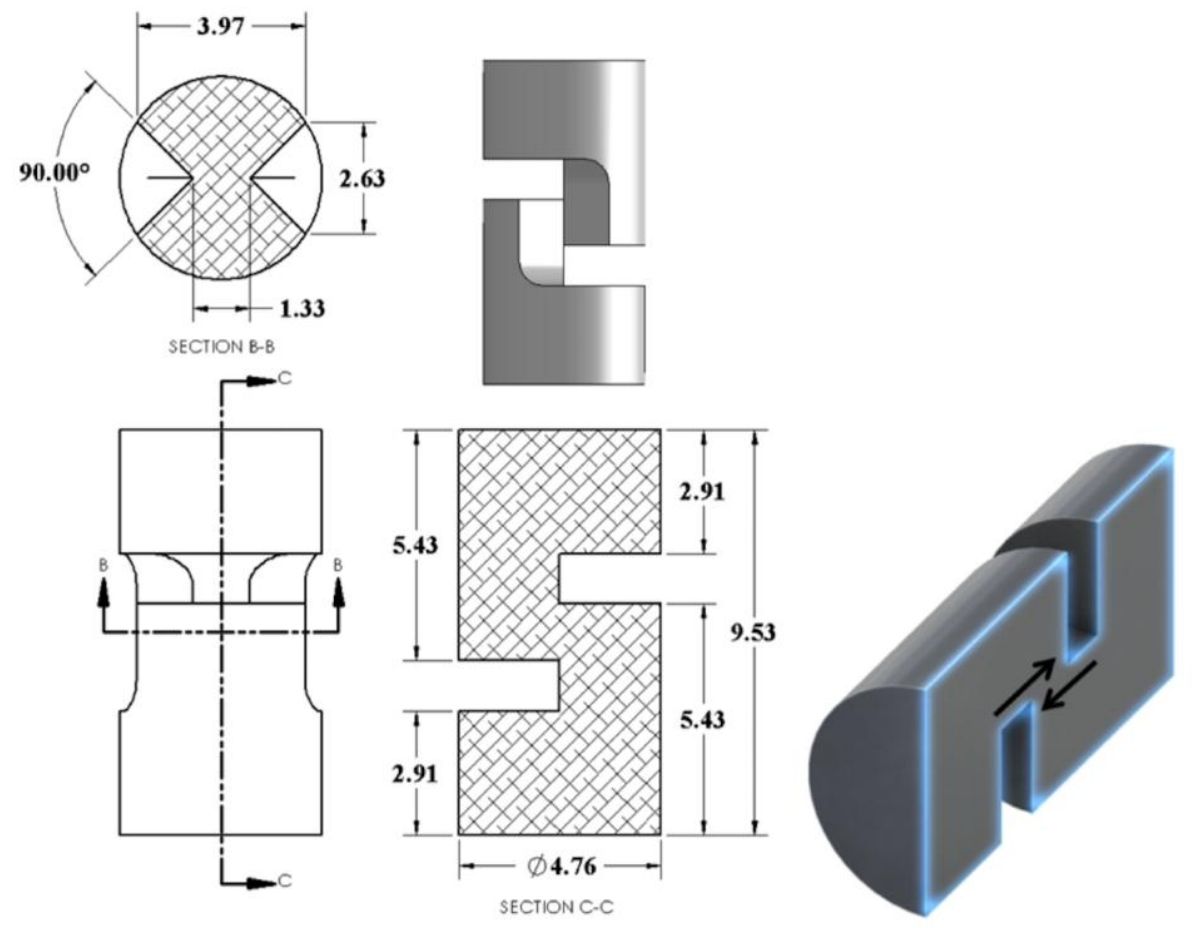


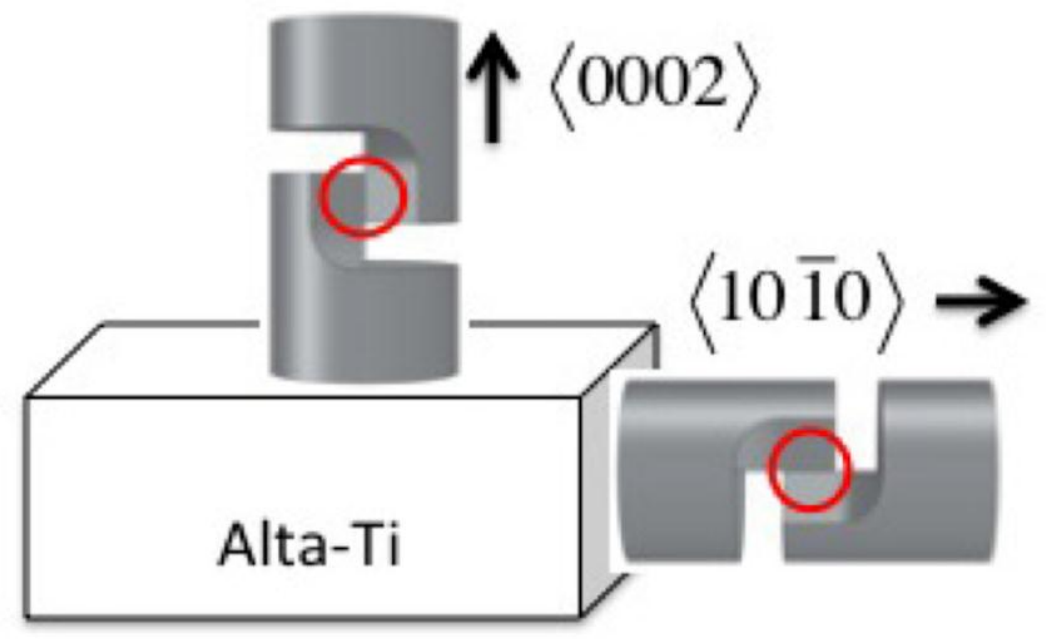




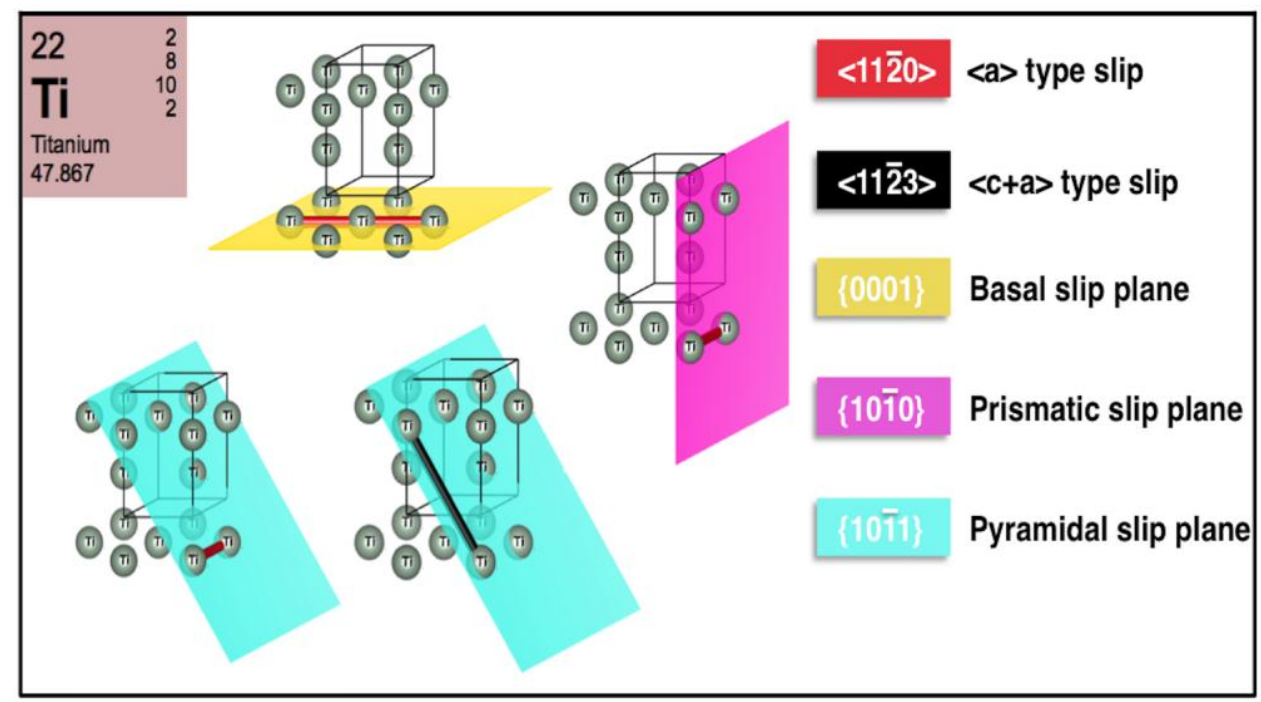




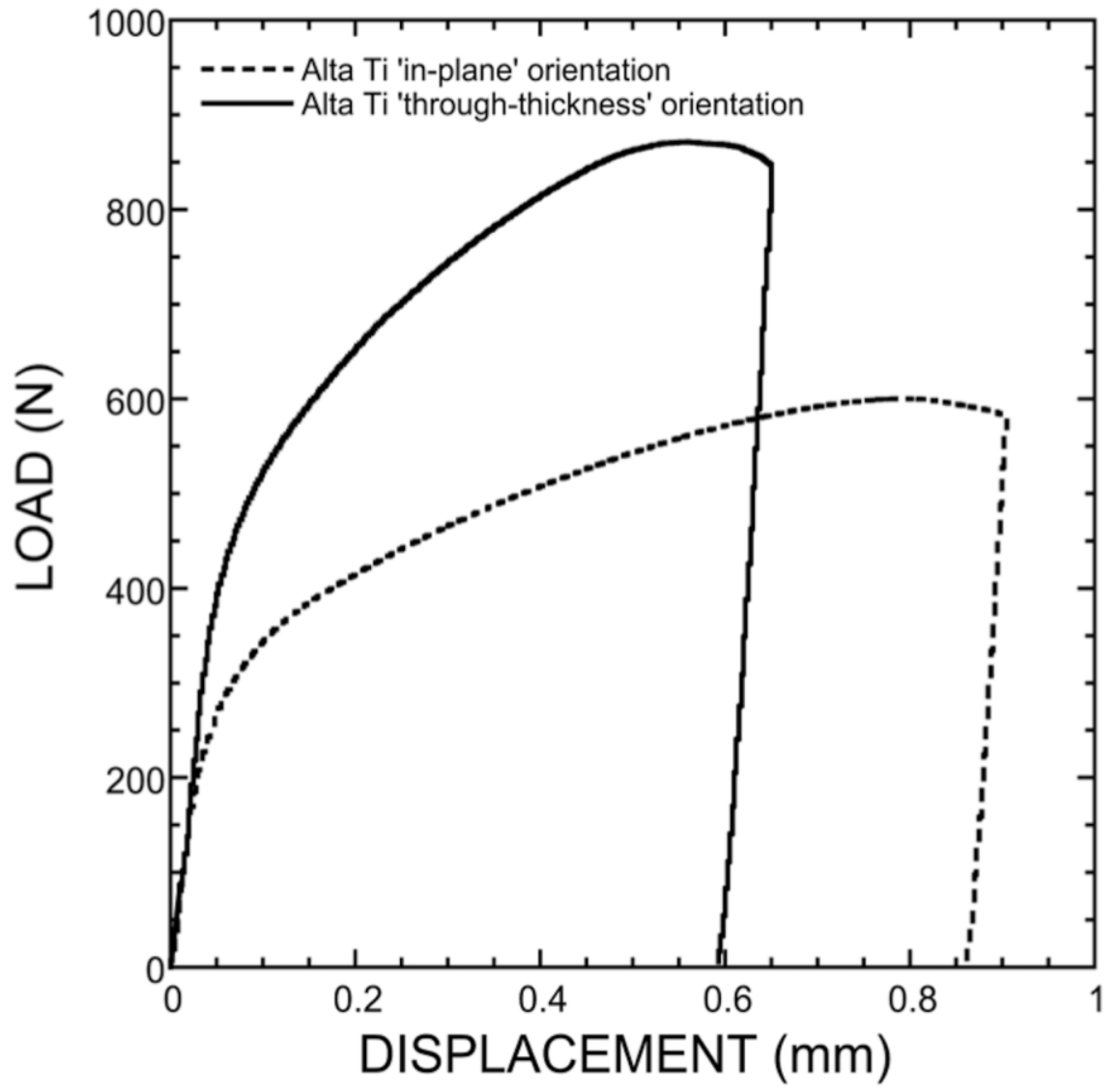



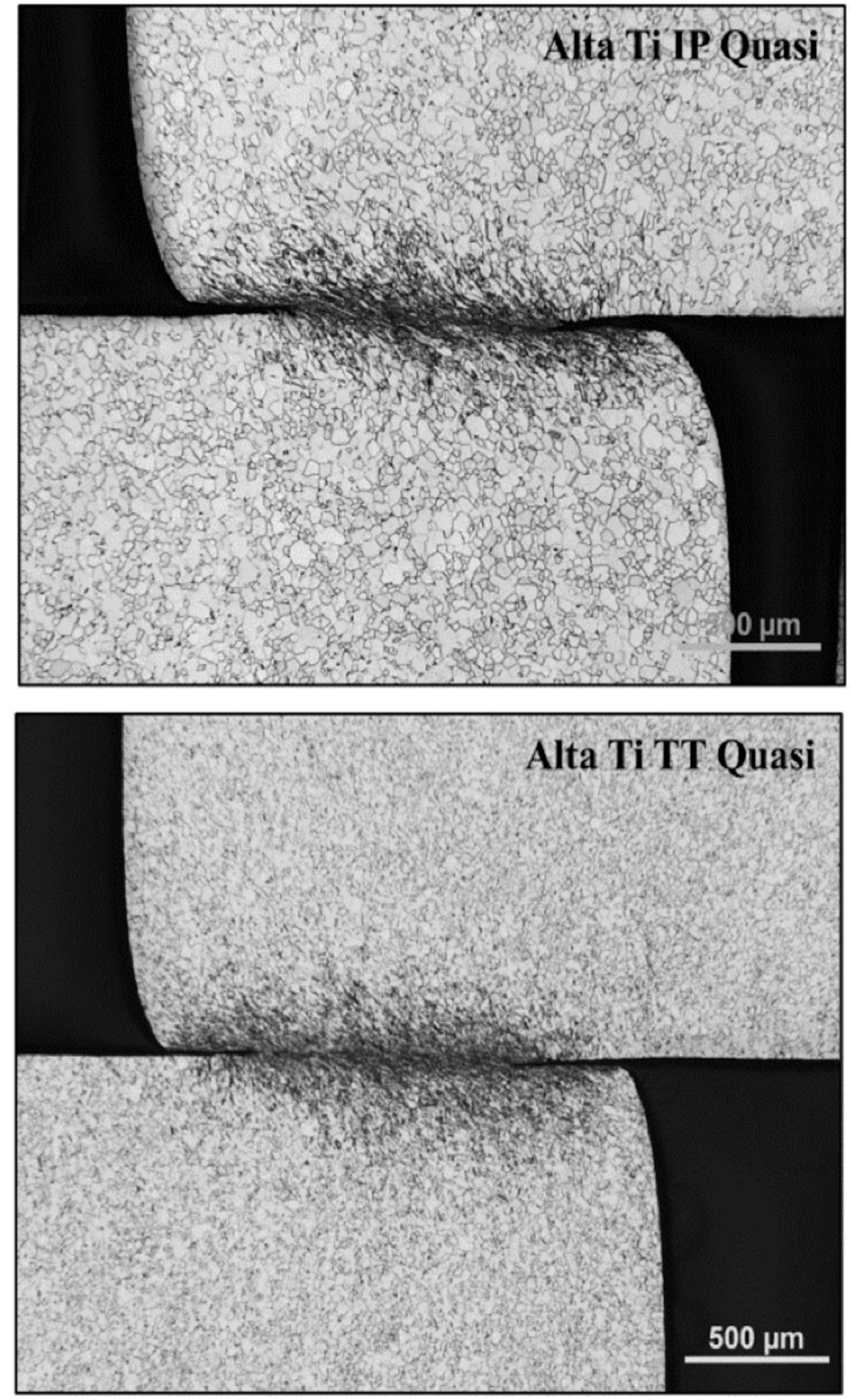

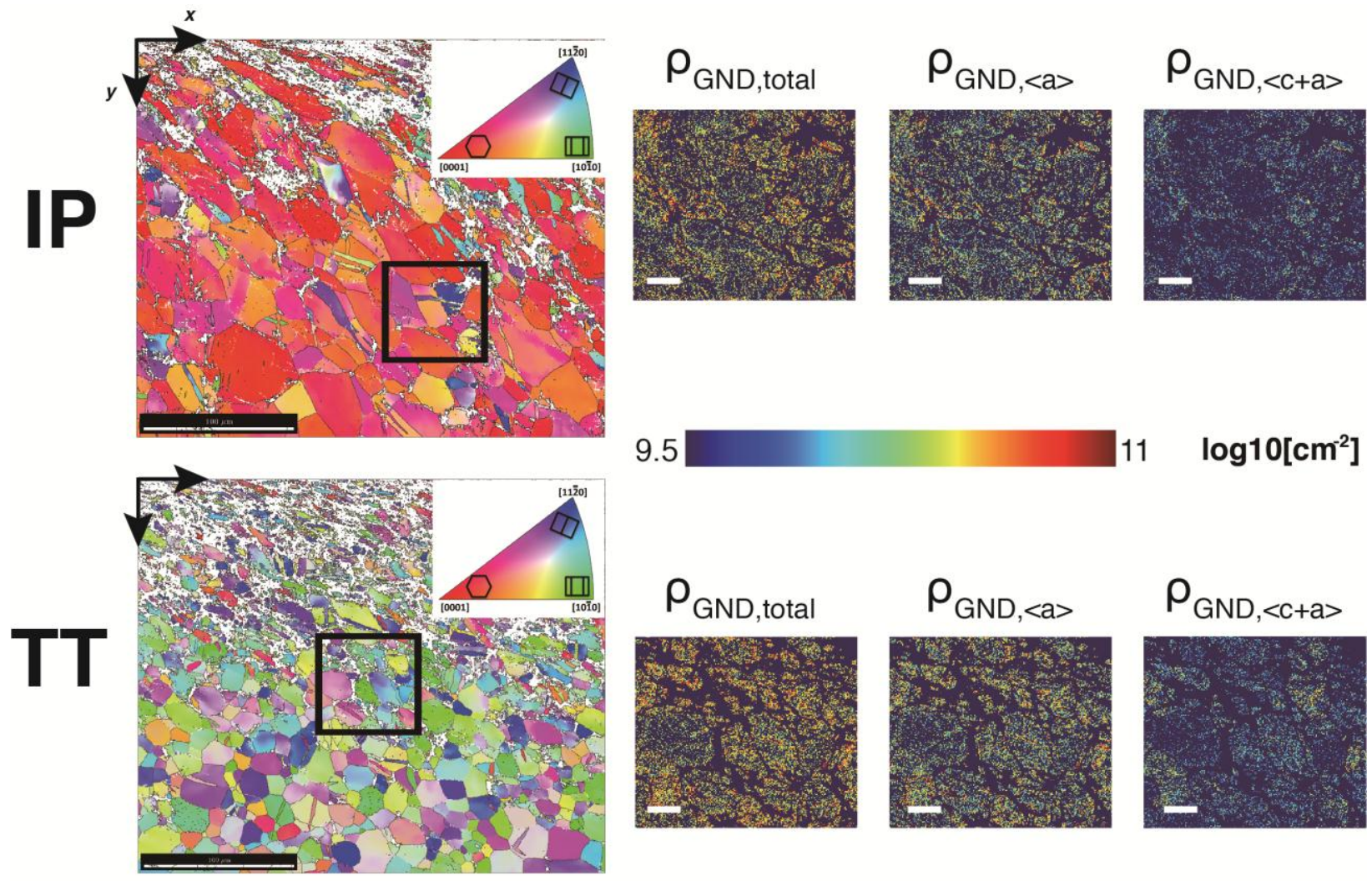

9.5

$11 \log 10\left[\mathrm{~cm}^{-2}\right]$

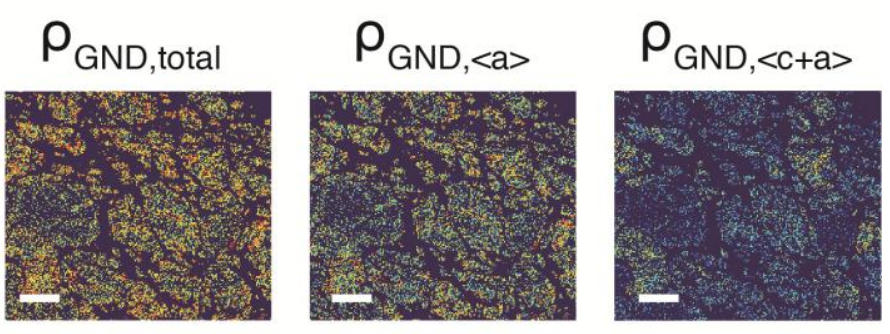




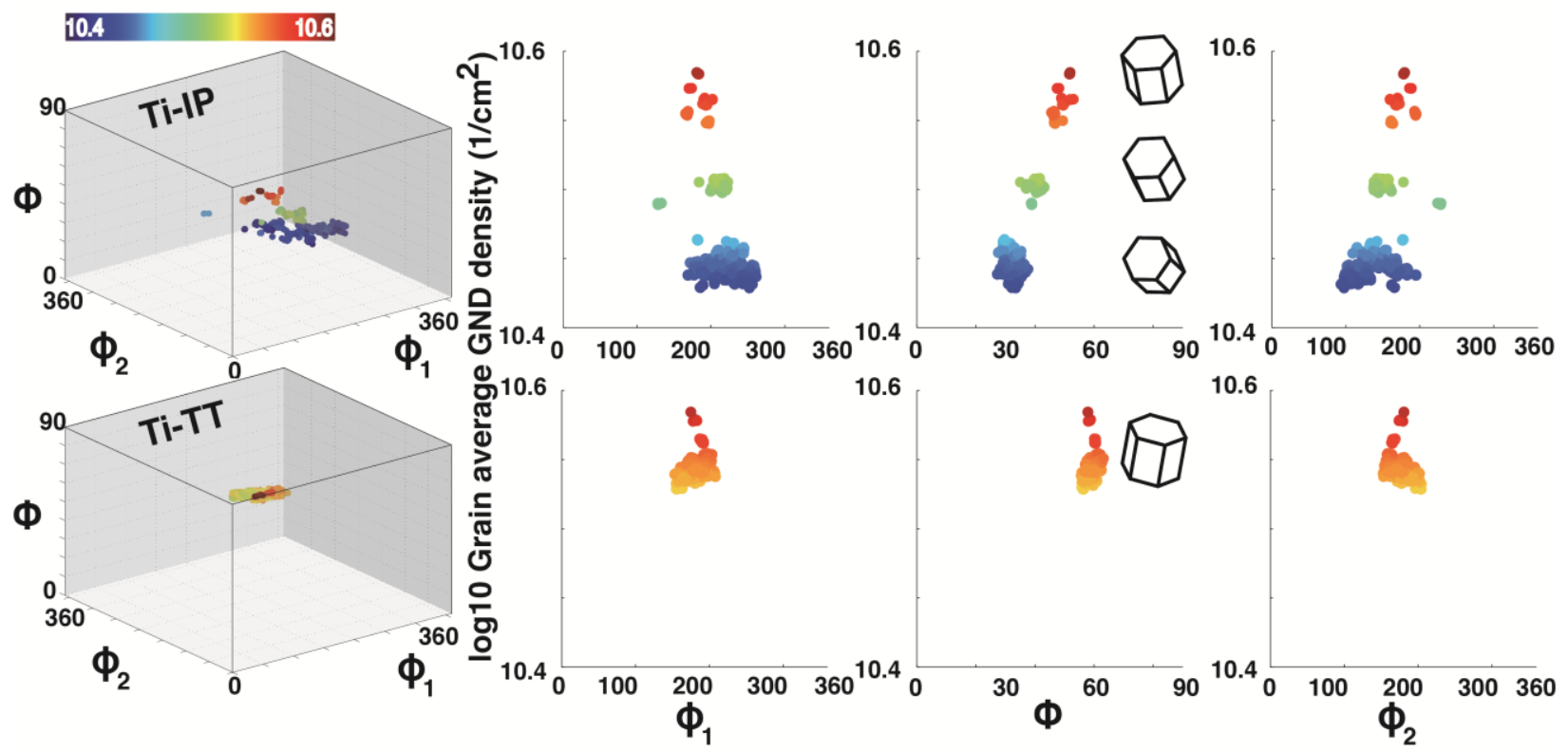




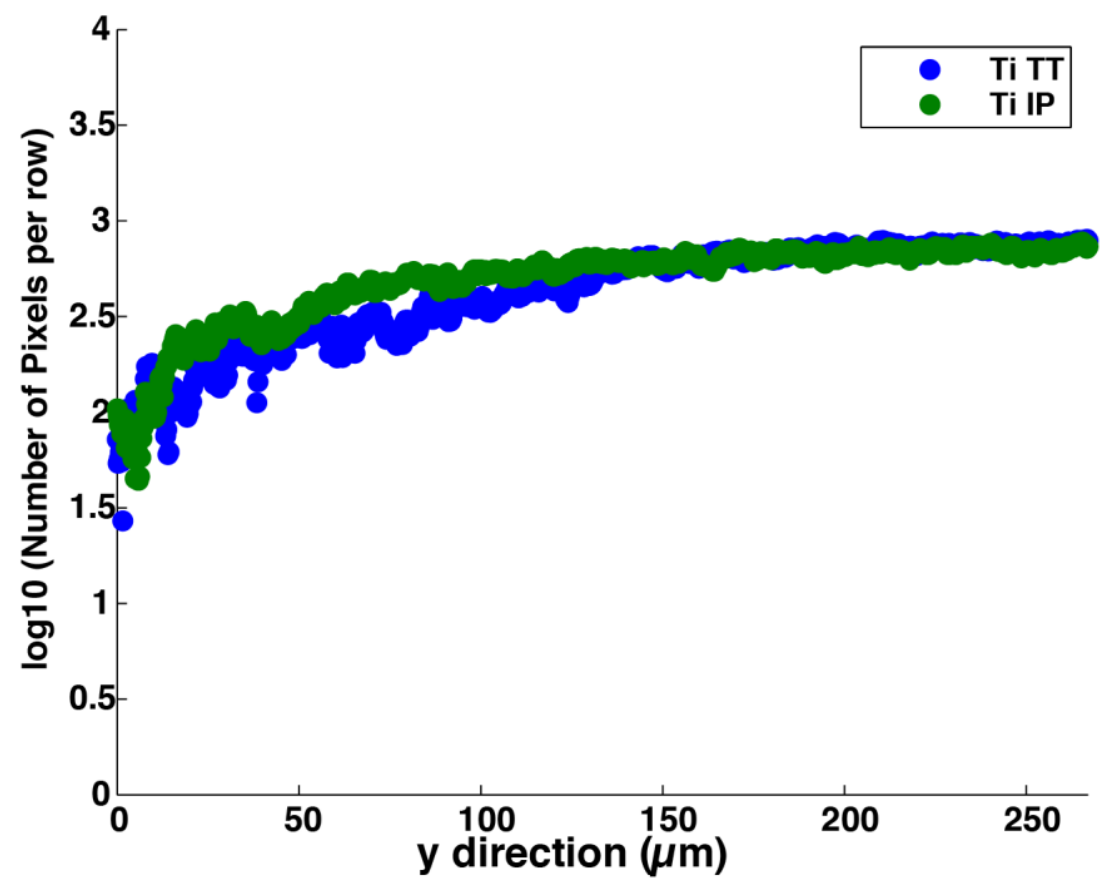



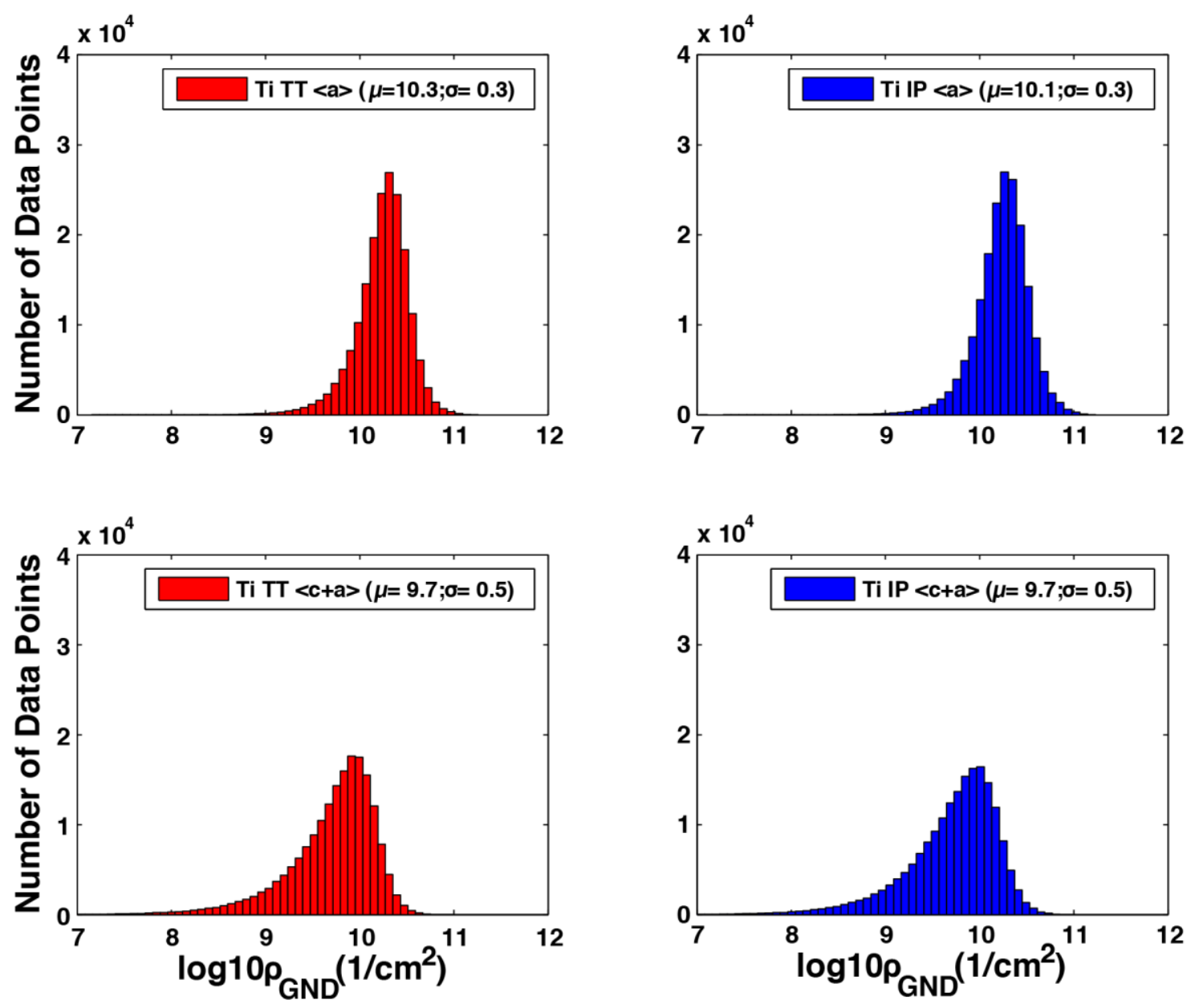

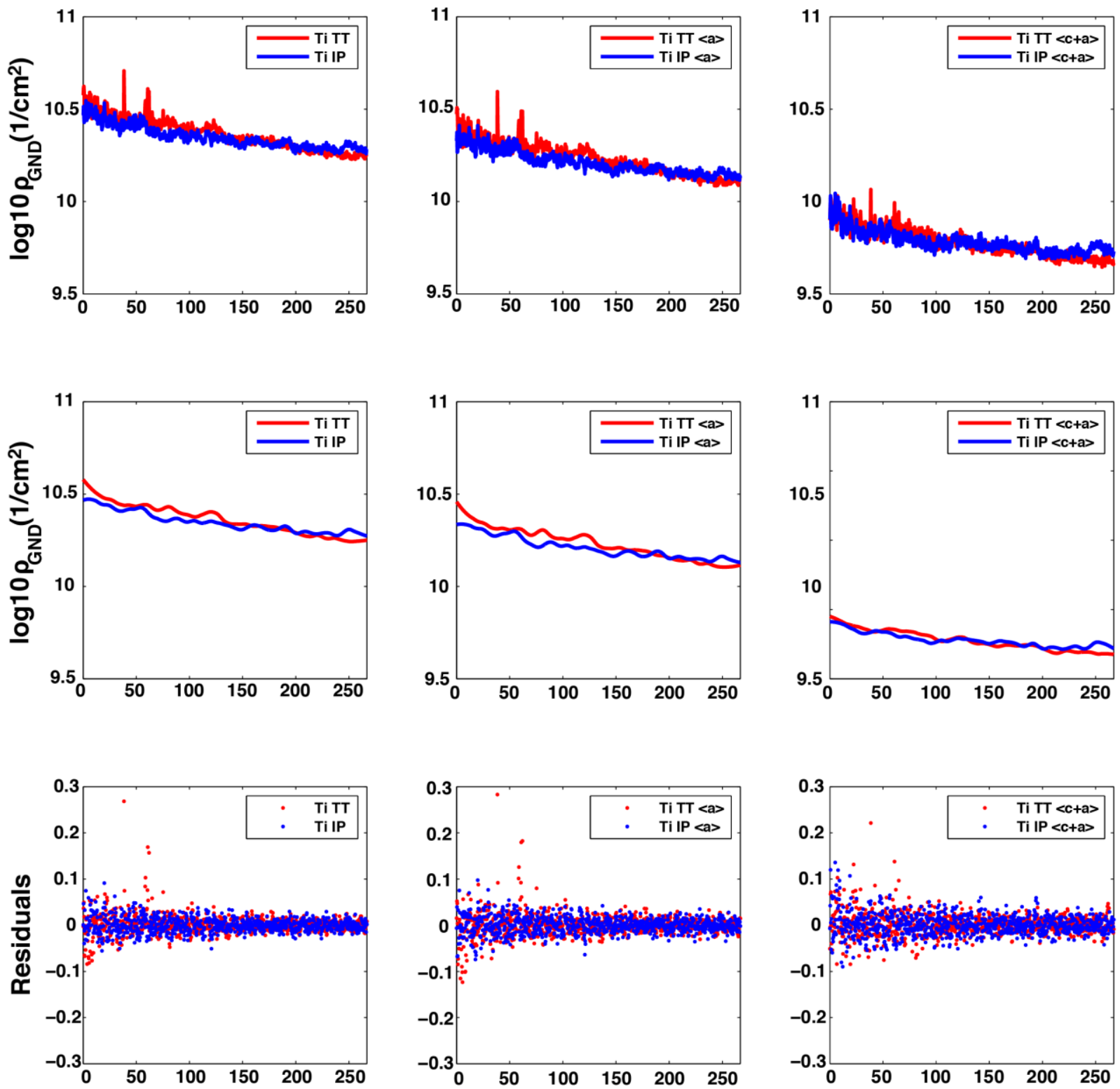